\title{
Das Prophetenbild in der modernen deutschen Literatur - ein interreligiöses Diskurs
}

\author{
Dr. Tarek Mohammed Abo El-meilla Mhani \\ Lektor an der deutschen Abteilung - Al- Alsun Fakultät - \\ Suez Kanal Universität .
}

\begin{abstract}
:
In diesem vorliegenden Beitrag werde ich eigentlich versuchen, ein deutsches interreligiöses Modell der westlichen Prophetenforschung $\mathrm{zu}$ rechtfertigen. Annemarie Schimmel repräsentiert eine unerschütterliche Quelle für eine kritische Verständigung des Islam. Sie hat die ethische Haltung des Propheten am schönsten ausgedrückt. Sein Menschentum tritt besonders in den Vordergrund. Durch die Menschheit des Propheten können die Muslimen ihre Hauptziele gut kennenlernen. Der Prophet lehrt, dass der Islam nicht die totale Abwertung der Welt verlangt. Der Mensch ist ein unweigerlicher Teil der Schöpfung deshalb respektiert der Islam die Würde aller Menschen. Am besten hat der Prophet den möglichen irdischen Mitteln bedient, um das Leben richtig zu genießen. Daraufhin dient das menschliche Leben des Proheten allen Muslimen als Vorbild. Dehalb gelang ihm, den Menschen seine Botschaft zu vermitteln.

Mehrmals hat Schimmel betont, dass der Islam für einen wahren Respekt der Frauen plädiert hat. Prinzipell weden die ganzen Rechte der Frauen im Islam garantiert. Das westliche Frauenbild im Islam hat Schimmel besonders motiviert, um das Richtige und Sachliche zu suchen. Sie hat sich durch ihr Buch"Meine Seele ist Frau" ganz für eine neue Formnug des islamischen Frauenbilds im Westen eingesetzt. - Das Leben des Propheten beinhaltet viele Hinweise auf die gute Behandlung seiner Frauen. Seine Liebe und Loyalität
\end{abstract}


gegenüber Hadiga haben das verzerrte westliche Frauenbild ausgeschlossen. Er hat sich auch von dem Leben seiner Familie nicht zurückgezogen. Immer half er ihnen bei der schweren Haushalt. Ausdrücklich hat er den all Muslimen ständig empfohlen, die Frauen menschlich und geduldig zu behandeln. Die Bedeutung der Mütter ist keineswegs im Islam ignoriert. Die Rücksichtnahme der Mütter ist für allen Muslimen bindend.

-Ich hoffe, dass der Zweck dieses Beitrags erfüllt wird. Die Intrreligiösität wird durch das Propetenbild von Schimmels Werke sachlich funktioniert. Sie hinterlässt kritische, ostwestliche Untersuchungen im Bezug auf Respekt vor der Menschenwürde. Immer unterstreicht sie die gegenseitigen Gemeinsamkeiten zwischen verschiedenen Farben, Kulturen und Religionen. Schimmel legt die absoluten Verschiedenheiten zwischen Kulturen beiseite. Es gelang ihr, einen wissenschaftlichen Weg zu den Annäherungen unterschiedlicher Völker und Nationen zu geben.

Interreligiösität - Das Prophetenbild - Annemarie Schimmel - Menschheit des Propheten - ToleranzInterkulturalität 
Das Prophetenbild in der modernen deutschen Literatur ein interreligiöses Diskurs

Dr. Tarek Mohammed Abo El-meilla Mhani

مجلة وادي النيل للاراسات والبحوث الإنسانية والاجتماعية والتربوية (مجلة علمية محكمة)

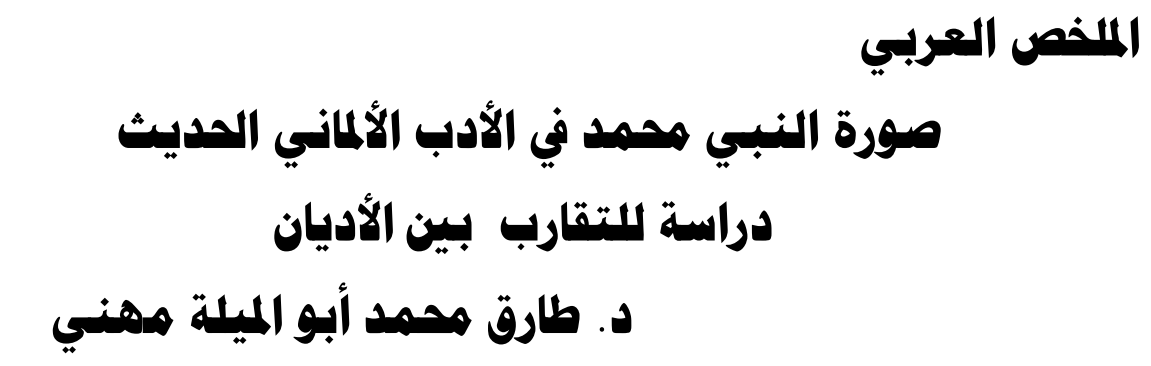

مدرس بكلية الألسن - قسم اللغة الألمانية

جامعة قناة السويس

تعد أنماري شيمل (1922-2003) من أبرز المفكرين الألمان في العصر

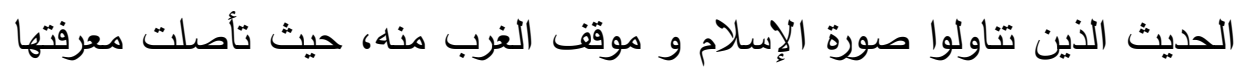

بقضايا الإسلام العامة والخاصة مثل: التصوف الإسلامي، والنبي محمد عليه

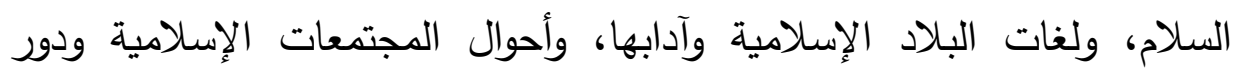

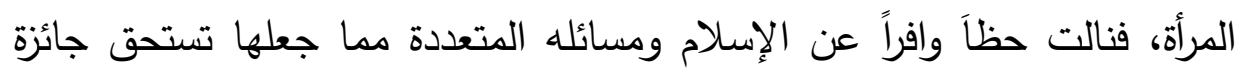
السلام الألمانية عام 1995 كأول مستشرقة ودراسة للإسلام.

يتناول هذا البحث صورة النبي محمد عليه السلام في أعمال المستشرقة

الألمانية أنماري شيمل، حيث قدمت نموذجاً علمياً يقوم على: الدارية

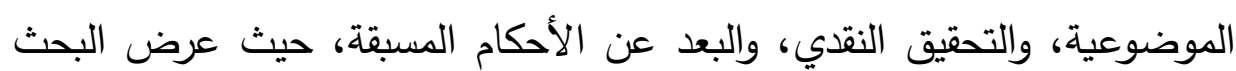

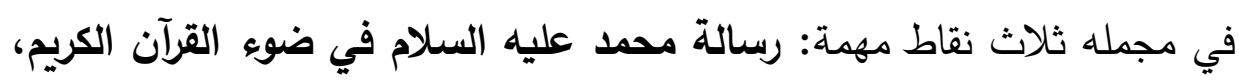
وتأسيس دولة إسلامية تقوم على الجمع بين الدين والدولة، والجانب الأخلاقي

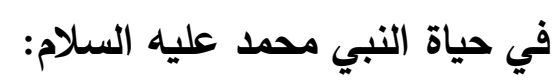

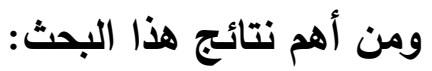

1- نجحت الكاتبة أن تبرز صورة النبي محمد عليه السلام من خلال المصادر الإسلامية ذات الثقة وأن تقدم للغرب والمسلمين كذلك إسهاماً علميا 
وموضوعيا يعتمد على الدراسة المتبصرة والتحقيق العقلي البناء لصورة نبي الإسلام عليه السلام، وهي بذلك تدحض جذور صورة الغرب العدائية للإسلام وتقطع على أصحاب الأحكام المسبقة حجتهم في هجومهم على الإسلام ونبيه الكريم.

2- ليس الإسلام شعائر وطقوسا مجردة، بل هو دين أقام دولة دينية ومدنية في آن واحد، جمعت بين صور التآخي بين المسلمين بعضهم البعض من ناحية وعقد المعاهدات والصلح مع أهل الكتاب من ناحية أخرى، وتأسيس الدولة سياسياً وعسكريا واجتماعياً وأخلاقياً وذلك في عصر النبوة والصحابة الكرام، وأكدت شيمل أن الإسلام يختلف في هذا الجانب عن الديانات الأخرى ولاسيما المسيحية في: الجمع بين قضايا الدين والدنيا وسعادة الفرد في الدنيا والآخرة والتوافق بين العقل والنقل.

\section{كلمات هفتاهية:}

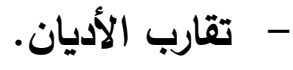

- - صورة النبي في الأدب الألماني.

$$
\text { - }
$$

- - جوانب الإسلام الموضوعية.

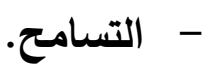

- - التواصل مع الآخر. - - - 
Das Prophetenbild in der modernen deutschen Literatur ein interreligiöses Diskurs

Dr. Tarek Mohammed Abo El-meilla Mhani

مجلة وادي النيل للاراسات والبحوث الإنسانية والاجتماعية و التربوية (مجلة علمية محكمة)

Das Prophetenbild in der modernen deutschen Literatur- ein interreligiöses Diskurs

Dr. Tarek Mohammed Abo El-meilla Mhani

Lektor an der deutschen Abteilung - Al- Alsun Fakultät -

Suez Kanal Universität .

\section{Vorüberlegungen}

In dem vorliegenden Beitrag werde ich eigentlich versuchen, ein deutsches interreligiöses Modell der westlichen Prophetenforschung $\mathrm{zu}$ rechtfertigen. Annemarie Schimmel repräsentiert eine unerschütterliche Quelle für eine kritische Verständigung des Islam. Daraufhin nimmt sie einen hervorragenden Platz - als zuverlässige Islamwissenschaftlerin und Orientalistin ein. Ein solcher Versuch könnte - darauf ziele ich vor allem - ein nachhaltiger Schritt zur besseren gegenseitigen Verständigung im Bereich des ost-westlichen , interreligiösen und interkulturellen Dialogs erreichen.Seit langer Zeit interessiere ich mich besonders für solchen Studien. (Vgl. dazu meine deutsche Diss."Ost-westliche Interkulturalität anhand der literarischen Werke von Goethe und Iqbal".)

Die Bedeutung Schimmels ist im arabischen Raum der Masse der Bevölkerung weniger bekannt. Ihre ständigen ostwestlichen Bemühungen sind auch kaum zu überschätzen. Wohl hat sie ein sachliches Verständnis des Islam besonders der Prophetenwirkung am schönsten ausgedrückt. Hier sind zwei Bücher von großer Bedeutung:

- Und Muhammad ist sein Prophet- eine Verehung des Propheten in der islamischen Frömmigkeit.

- Muhammad. (S. Literaturverzeichnis des beiliegenden Beitrags). 
مجلة وادي النيل للاراسات والبحوث الإنسانية و الاجتماعية والتربوية (مجلة علمية محكمة)

(ISSN : 2536 - 9555)

Mithilfe der beiden Bücher kann ich meinen Weg einschlagen, um dieser vorliegende Beitrag wissentlich $\mathrm{zu}$ gestalten. Sie enthalten eine detaillierte Darstellung des Propheten Muhammad. Es gelang ihr, den Propheten interreligiös $\mathrm{zu}$ lesen. Sie hat auch ganz für eine unvoreingenommene Behandlung der Botschaft des Propheten im islamischen Gedankengut eingesetzt. Die verschiedenen Züge der Größe und Weisheit des Propheten wurden hervorgehoben. Das hat mich besonders motiviert, dieses sachliche Prophetenbild zu thematisieren. Es soll hier noch einmal erwähnt werden, dass der vorliegende Beitrag mit meinem großem Interesse am ost-westlichen interkulturellen und interreligiösen Dialog zusammenhängt.

\section{Themenauswahl}

Es gibt eine Reihe von Gründen, die dazu geführt haben, dass ich die deutschen Islamwissenschaftlerin und Orientalistin Schimmel näher kennen lerne. Ich möchte kritisch beweisen, dass es in Europa und vor allem in Deutschland auch sachliche und neutrale Untersuchungen der Prophetenforschung gibt. Jetzt ist das westliche Prophetenbild nicht total schwarz. Die zuverlässige deutsche Übersetzerin fühlte sich für das tradierte Prophetenbild verantwortlich. Gelegentlich übte sie eine harsche Kritik daran. Zwar wurde dieses Bild durch Schimmel richtig erwidert. Dehalb habe ich ein bestimmtes Ziel verfolgt, die unentwegten Bemühungen von Schimmel für ein neues Prophetenbild im Westen zu entlarven.

"Von früh an hat mich das Unverständnis bekümmert, mit dem die westliche Welt der Gestalt des Propheten Muhammad gegenüberstand- nicht nur der Durchschnittsbürger, sondern auch viele Orientalisten, die in historischer Kritik dazu neigten, die negativen Qualitäten Muhammads herauszuarbeiten. Dazu zählen auch jene mittelalterlichen 
Das Prophetenbild in der modernen deutschen Literatur ein interreligiöses Diskurs

Dr. Tarek Mohammed Abo El-meilla Mhani

مجلة وادي النيل للاراسات والبحوث الإنسانية والاجتماعية والتربوية (مجلة علمية محكمة)

Fabeln, in denen der das Abendland und die christliche Kultur bedrohende Islam verzerrt dargestellt wurde."

Das negative Prophetendbild wurde im europäischen Selbstbewußt fest verankert. Es erstreckte sich vom Mittelalter bis in unsere Tage. Deshalb übernahm Schimmel eine schwere Botschaft, den Islam sowie das westliche Prophetenbild besser verstehen zu können. Im Laufe der Tage und Jahre besaß sie eine nachhaltige Rolle der ost-westlichen Vermittlung und auch ein fundiertes islamisches Wissen in Bezug auf die meisten strittigen islamischen Fragen und war ganz bereit, ihre Meinungen unumwunden $\mathrm{zu}$ erhellen. Mit den meisten Werken von Schimmel hat sie einen richtigen Weg für ein besseres Verständnis der Prophetenforschung im Westen sowie im Osten eingeschlagen. Durch die oben erwähnten Beiträge war sie im Stande, eine neue Gestalt des Propheten darzustellen.

"Er, der letzte in der langen, bei Adam beginnenden Kette der Propheten, brachte die abschließende Offenbarung, die in sich die Offenbarungen aller früheren Propheten einschlo $\beta$ und sie zu ihrer ursprünglichen Reinheit zurück führte."2

Es war die Zeit, wo das Bedürnis der Menschen nach einem Propheten herrschte, den letzten Prophten, als Gande für die Menschen.

Nachdem ich SchimmelsBuch "Und Muhammad ist Sein Prophet" gelesen hatte, wurde mir ganz klar, dass es uns eigentlich eine Gesamtdarstellung von Schimmels Affinität zum Islam anbietet, vor allem ihre unerschütterliche

${ }^{1}$ Schimmel, A. :Muhammad. Die Deutsche Bibliothek, München 2000, S. 7.

${ }^{2}$ Schimmel. Und Muhammad ist Sein Prophet - die Verehrung des Prophete in der islamischen Frömmigkeit, München 1989. S.8. 
مجلة وادي النيل للار اسات و البحوث الإنسانية والاجتماعية والتربوية (مجلة علمية محكمة)

(ISSN : 2536 - 9555)

Beschäftigung mit der Pophetenforschung und ihr Verhältnis zur ost-westlichen Interkulturalität.

Hier würde der Leser einige Fragen stellen: Weshalb hat sich Schimmel, als Wissenschaftlerin mit dem Islam und vor allem mit dem Propheten Muhammad beschäftgt? Was reizte Schimmel an der Persönlichkeit des Propheten? Kann Schimmel wohl den Propheten interreligiös lesen? Solche Fragen werden uns letztlich zu einer weiteren Frage führen, die besonders das Ziel des vorliegenden Beitrags darstellt, nämlich: Was ist eigentlich das Bild des Propheten im Werk von Annemarie Schimmel.

Um das sämtliche Bild des Propheten Muhammad im Werk von Schimmels erhellen zu können, werde ich die folgenden Punkte untersuchen:

1- Die Botschaft des Propheten Muhammad im Rahmen des Korans.

2- Die Begründung eines islamischen Staates.

3- Die ethische Haltung des Propheten Muhammad.

4- Ergebnisse.

\section{1- Die Botschaft des Propheten im Rahmen des Korans:}

Wenn ein solcher Forscher den Koran näher verstehen will, ist es notwendig und unweigerlich, auch von dem Propheten zu reden. Hier könne man auf den Koran als die erste und ehrlichste Grundlage für die Muhammads Botschaft nicht verzichten. So ist es wichtig, dass ich die engste Affinität zwischen dem Koran und dem Propheten Muhammad kennenlerne,um das ganze Bild des Propheten im Islam zu unterstreichen. Monotheismus ist auch die erste Säule der Botschaft des Propheten Muhammad.

"Sicher ist der Islam nicht nur eine Religion des Monotheismus, sondern auch der Einheit. Der Ausdruck Monotheismus hat einzige Bedeutung erlangt, über die hinaus nchts geht, nämlich der Anspruch, dass es nur einen Gott gibt, 
Das Prophetenbild in der modernen deutschen Literatur ein interreligiöses Diskurs

Dr. Tarek Mohammed Abo El-meilla Mhani

مجلة وادي النيل للاراسات والبحوث الإنسانية والاجتماعية والتربوية (مجلة علمية محكمة)

der Himmel und Erde und alles was zwischen liegt geschaffen hat und der alles wieder zu sich nehmen kann. Diese Bedeutung ist gegen die Auffassung, dass es zwei oder mehrere Götter gäbe. Der Islam beruht nicht nur auf dem Glauben, an die Einheit Gottes, sondern auf allem, was das Göttliche, Politische, Soziale und alle anderen Sphären des menschlichen Daseins in dieser Welt umfasst."

Wir finden das Hauptanliegen des Korans die Herstellung des Gottesverehrung von dem Monotheismus durch das praktische Leben des Propheten. Prinzipell muss der monotheistische Glaube fest verankert werden, um die Bedeutung der Botschaft des Propheten gut kennenzulernen.

"Gleichwohl enthält der Koran eine Fülle von Material, das historisch von großer Bedeutung ist und mit dessen Hilfe man nicht nur Rückschlüsse auf die sozialen und religiösen Verhätlnisse in Mekka und Medina zur Zeit Mohammads ziehen kann, sondern auch auf Muhammads Wirken als Prophet. ${ }^{\prime 4}$

Schimmel weiste mehrmals auf den Wert des Monotheismus im Islam hin. Daraufhin glaubte sie, dass der Prophet der richtige Träger der Gottesverehrung durch den Monotheismus war." Der eine Islam ist durch das Glaubensbekenntnis,den absoluten Monotheismus und den Glauben an die abschließende Rolle des Propheten Muhammad gekennzeichnet. ${ }^{\prime 5}$

Die Botschaft des Propheten beruhte sich im Wesentlichen auf dem Monotheismus. Deshalb brauchte der Prophet

3 Mousse, M. Youssef: Islam und dessen Notwendigkeit für die Menschheit, übersetzt v. Elisabeth Khorshed, Al- Ahram Druck, Kalioub 2004,.S, 30.

4 Bobzin, Hartmut: Mohammed,Verlag C.H.Beck oHG, München 2002,S. 41.

${ }^{5}$ Schimmel. Spiegelungen des Islam, Berlin: Ed.q,2002,S.67. 
مجلة وادي النيل للاراسات والبحوث الإنسانية والاجتماعية والتربوية (مجلة علمية محكمة)

(ISSN : 2536 - 9555)

unerschütterliche Geduld, um den Mekkanern von der neuen Gottesvorstellung zu überzeugen. Der Prophet beschwor die Einwoher Mekkas, sich von der Vielgötterei anzuwenden und nur noch den Einen und Wahren Gott anzubeten.Deshalb war der Prophet zeitlebens ein wahrer Monotheist und Überbringer dieses Glaubens an allen Menschen.

"Der im siebten Jahrhundert der christlichen Zeichtrechnung entstandene Islam hat seinen Ursprung in der göttlichen Offenbarung des Propheten Muhammad. Seit den Anfängen des Islam sind die Muslime zu dem Glaubensbekenntnis angehalten, dass Allah der einzige und ausschließliche Gott ist, der die Welt erschaffen hat und die Menschen für ihre verübten und unterlassenen Taten am Ende aller Tage beurteilen wird."

Die meisten Werke von Schimmel enthielten viele Hinweise auf Monotheismus als Ursprung des Islam und der Prophet war die praktische Prüfung dafür."Muhammad stellt die Grenze bei der Definition des Islam dar und trennt ihn von allen anderen Glaubensformen. ${ }^{17}$

Seit Aufkommen des Islam gilt er als die einzige Religion des wahren Monotheismus, für den der Prophet sein zeitlebens plädiert hat.

"Die Botschaft, die Muhammed ab 610 erhielt, sprach von Gott als dem Schöpfer und Richter, der die Menschen eines Tages vor seinen Richterstuhl fordern wird. Wenn sie sich nicht den Geboten der Nächstenliebe Gerechtigkeit und Ehrlichkeit unterwerfen." 8

Schimmel respektierte besonders die Einheit Gottes im Islam. Das bezeugte ihre richtige Verständigung der islamischen Lehren und Glauben. Sie war davon ganz

${ }^{6}$ Ebd.S.39.

${ }^{7}$ Schimmel: Mystische Dimensionen des Islam, Frankfurt am Main u.a. 1995,S 304.

${ }^{8}$ Schimmel: Muhammed, S.12 
Das Prophetenbild in der modernen deutschen Literatur ein interreligiöses Diskurs

Dr. Tarek Mohammed Abo El-meilla Mhani

مجلة وادي النيل للاراسات والبحوث الإنسانية والاجتماعية والتربوية (مجلة علمية محكمة)

überzeugt, dass die Verschiedenheit zwischen Islam undChristentum hauptsächlich im Konzept der Gottesvorstellunglag.

"Das beherrschende Thema des Koran ist der strikte Monotheismus, der Glaube an den Einen Gott; das kommt am prägnantesten in Sura 112, dem Bekenntnis zur Einheit Gottes Tauhid, zum Ausdruck. In der abendländischen Sicht des Islam wurde Sura 112 ganz überwiegend als Kampfansage gegen die Christen und ihren Glauben an die Gottessohnschaft Jesu verstanden." ${ }^{\prime \prime}$

Für Schimmel hatte die Interreligiösität zwischen Islam und Christentum eine enge Affinität zum Gottesvorstellung. "In den Suren des Korans ist die Verkündigung des Propheten noch nicht eigentlich von dem Gedanken getragen, dass es nur einen Gott gibt. Eine monotheistische Gottesvorstellung liegt hier erst im Ansatz vor. Dagegn spielt die Polemik gegen die Vielgötterei eine bedeutende Rolle in den koranischen Propheten- und Straflegenden."10

Schimmel hat den Monotheisums im Islam im Wesentlichen ausgewirkt, um die maßgebliche Rolle des Propheten Muhammad als Überbringer der monotheistischen Religion zu veranschaulichen. Die Einheit Gottes ist für jeden Muslimen ganz bindend. Daraufhin ist der Islam von dem Judentum und Christentum in Bezug auf Gottesauffassung ganz verschieden obwohl die drei geoffenbarten Religionen zum reinen Monotheismus rufen.

"In dem zweiteiligen Glaubensbekenntnis; Es gibt keine Gottheit außer Gott, und Muhammad ist der Gesandte Gottes stellt die zweite Hälfte, die den Islam gewissermaßen zu einer

\footnotetext{
${ }^{9}$ Bobzin, Hartmut: Der Koran,C.H.Beck, München 2007, S 57.

${ }^{10}$ Paret, Rudi: Mohammed und der Koran,W.Kohlhammer,neunte Auf., Stuttgart 2005, S.103.
} 
مجلة وادي النيل للاراسات والبحوث الإنسانية والاجتماعية والتربوية (مجلة علمية محكمة)

(ISSN : 2536 - 9555)

scharf definierten Religion macht,eine Aussage über Gottes Aktivität dar: durch die Sendung Seines Propheten offenbart sich Gott der Welt."11

Deswegen wurde Muhammads Sunna, seine Lebensweise zur Richtschnur für die Muslime.Der Prophet fand in den koranischen Aussagen Geduld, Trost und Vorbild. Auf der anderen Seite muss man nicht vergessen, dass Monotheismus die richtige Gottesfassung von Adam bis zum Propheten Muhammad ist. Der reine Monotheismus ist die interreligiöse Gemeinsamkeit zwischen den geoffenbarten Religionen.

"Die koranischen Offenbarungen hatten nie aufgehört. Während sie zu Beginn von Muhammeds Laufbahn von der den Schrecken des Gerichtes, von der Allmacht des einen und einzigen anbetungswürdigen Gottes sprechen, enthalten sie in der mittleren Krisenperiode viele Hinweise auf die Leiden früherer Propheten, die dazu dienen sollen, Muhammed selbst in seinem Kampf zu stärken." 12

Die unentwegten Bemühungen des Propheten Muhammad haben Schimmel besonders bezaubert. Sie führte den Islam vor allem auf den Monotheismus zurück. Die Mekkaner lehnten diesen islamischen Glauben kategorisch ab. Deshalb wurde der Prophet von ihrer Sturrheit und Verstockheit streng heimgesucht. Unbeirrbar hat er bei ihnen lange ausgeharrt, um seine monotheistische Botschaft $\mathrm{zu}$ vermitteln. Und damit erwies der Prophet der Menschheit enorme Verdienste. Mit Recht konnte er ausdrücklich sein, eine ganze Nation zum Lichte der Wahrheit bekehrt zu haben.

\section{2-Die Begründung des islamischen Staates}

Wie der Prophet einen islamischen Staat gegründet hat, ist eine wichtige Frage?Ausführlich hat Schimmel diesen Punkt

${ }^{11}$ Schimmel: Und Mohammed ist Sein Prophet, S.23.

${ }^{12}$ Ebd. S.15. 
Das Prophetenbild in der modernen deutschen Literatur ein interreligiöses Diskurs

Dr. Tarek Mohammed Abo El-meilla Mhani

مجلة وادي النيل للاراسات والبحوث الإنسانية والاجتماعية و التربوية (مجلة علمية محكمة)

angemessen darlegt. Sie zeigte besonders die politische Rolle des Propheten in Medina, um eine einheitliche Gemeinde (Umma) aufzurichten.

"Demjenigen, der Religion und Politik säuber voneinander $z u$ trennen versucht und Religion als private Angelegenheit ansieht, wird erklärt, dass Religion und Staat wie die beiden Seiten einer Münze zusammengehören. Muhammads staatsmännische Klugheit, seine sozialen Verbesserung in Mekka und Madina, seine als Muster moderner demokratischer Einrichtungen gepriesene Gemeindeordnung von Medina, seine klug Wirtschaftsführung werden modernen Muslim als Vorbild hingestellt, nach dem er sein gestalten soll."13

Die Rolle des Propheten wird hier politisch gefärbt. Er hat sich im höchsten Maße für eine Duchsetzung des islamischen Staates eingesetzt. Es gelang ihm, zwischen Politik und Religion richtig zu verschmelzen.

"Es erschient ihm überzeugend, dass ein erfolgreicher Prophet seine von Gott empfangne Botschaft auch auf Erden mit irdischen Mitteln ausbreitet. Der scheinbare Widerspruch zwischen Prophet und Staatsmann ist nach seiner Aufffassung gerade ein Zeichen für die allumfassende Leistung des Propheten, der Beweis für die Größe und Wahrheit seiner Botschaft." "14

Die Medinas Bewölkerungen waren kulturell und religiös unterschiedlich. Zuerst wurden diese verschiedenen Varianten interkulturell und interreligiös abgesagt. Das war die erste sowie dringlichste Aufgabe des Propheten in Medina.

"Der Prophet muss aber ein beachtliches politisches Geschick bewiesen haben, da er nach relativ kurzer Zeit schon

${ }^{13}$ Schimmel. Muhammad. S.33.

${ }^{14}$ Ebd.46. 
einige Fortschritte in der Islamisierung Madinas durch eine freundliche Überzeugungsarbeit verwirklicht hatte. Den ersten Schritt dazu hatte er damit erreicht, dass er die mit ihm Ausgewanderten in der neuen Umgebung heimisch machte. Das geschah auf die Weise, dass der Bruderschaftsbeziehungen herstellte zwischen den Neuzuwandern und den bereits in der Stadt vorhandenen Muslims. Das zielte vor allem darauf ab, die weitgehend mittellosen Auswanderer dadurch in ihrer materiellen Existenz zu sichern." 15

Es gelang dem Propheten, eine interreligiöse Umgebung in Medina dazu stellen. Er hat sich für eine sachliche Brüderlichkeit zwischen den Muslimen einerseits und für den Frieden mit den anderen medinensichen Nicht-Muslimen andererseits eingesetzt. Der Prophet war auch im stande, die Dispute und Kriege in Medina zu schlichten.

"Muhammad traf in Medina die vor ihm Ausgewanderten sowie auf eine Gemeinde von Gläubigen, die sich schon gebildet hatte, während er noch von Mekka aus seinen Glauben verkündigte. Aber auch diejenigen unter den arabischen Stämmen Medinas, die sich noch nicht zu Muhammad bekannten, hießen willkommen, weil sie sich vom ihm die Schlichtung der erbitterten Feindschaft erhofften, die jahrlang unter ihnen geherrscht und das Leben in der Ansiedlung lahmgelegt hatte. Tatsächlich gewann Muhammad binnen kurzer Zeit eine so große Autorität in Medina, dass sich alle arabischen Bewohner der Stadt zum neuen Glauben bekannten."16

15 Serauky, Eberhard. Geschichte des Islam- Enstehung, Entwicklung und Wirkung von den Anfängen bis zur Mitte des 20. Jahrhunderts., Gulde Druck GmbH, Tübingen 2003, S.88.

${ }^{16}$ Der Koran-:- in der Übersetzung von Friedrich Rückert, hrsg. Von Hartmut Bobzin mit erklärenden Anmerkungen von Wolfdietrich Fischer, Ergon Verlag,Würzburg 1995. S.491. 
Das Prophetenbild in der modernen deutschen Literatur ein interreligiöses Diskurs

Dr. Tarek Mohammed Abo El-meilla Mhani

مجلة وادي النيل للاراسات والبحوث الإنسانية والاجتماعية والتربوية (مجلة علمية محكمة)

Hier gewann der Prophet die Gelegenheit, um seine Botschaft ausdrücklich $\mathrm{zu}$ vermitteln. In Medina fand Muhammad die Rahmenbedinungen ganz anders als in Mekka. Er hatte seinen Weg erfolgreich eingeschlagen, um einen islamischen Staat systematisch zu begründen.

"Damit schuf der Prophet eine neue Solidaritätsgemeinschaft auf religiöser Grundlage. Sie wuchs rasch, wobei von früher Stunde an ein verändertes Konversionsmuster zu beachten war". 17

"Indem Muhammad in Medina zum Oberhaupt einer Gemeinschaft geworden war, die er nicht nur religiös zu unterweisen, sondern auch in allen inneren und äußeren Angelegenheiten $z u$ leiten hatte, musste er das Zusammenleben mit Menschen ordnen und durch gesetzliche Bestimmungen regeln. Auch das Zusammenleben mit Juden gab Anlaß genug, der muslimischen Gemeinde ein Gesetz zu geben, das dem mosaischen Gesetz vergleichbar hatte."18

Der Prophet hat einen historischen interreligiösen und vor allem menschlichen Vertrag mit den medinensischen Juden und Christen geschlossen. Er suchte nur den Frieden mit ihnen. Seine religiöseRechte wurden im höchten Maße garantiert. Die Werte der Gleichheit und Gerechtigkeit zwischen den Muslimen und den Juden und Christen wurden auch besonders verankert, weildie Botschaft des Propheten mit dem der früheren Offenbarungsreligionen vereinbart hatte.

"Ausdrücklich werden die Juden in dieses neue Zusammenleben eingeschlossen, wenn sie sich den genannten Gemeinschaftsprinzipien anschließen. Hier waren wichtige neue Ansätze für ein besseres gegenseitiges Verständnis

${ }^{17}$ Krämer, Grunden. Geschichte des Islam.Verlag C.H. Beck, München 2005.S.21.

${ }^{18}$ Der Koran:- in der Übersetzung von Friedrich Rückert, S.495.

$47)$ 
مجلة وادي النيل للاراسات والبحوث الإنسانية والاجتماعية والتربوية (مجلة علمية محكمة)

(ISSN : 2536 - 9555)

erkennbar; auch wurde damit die gemeinsame Verantwortung für die Friedensbewahrung auf alle Bewohner ausgedehnt. Muhammad brachte mit diesen Gedanken seine tiefe Überzeugung von notwendigen Kooperation mit den jüdischen Gläubigen zum Ausdruck."19

Nun stand der Prophet nach langen Bemühungen an der Spitze einer großen muslimischen Gemeinde, die mit den anderen medinensischen Bewohnern interkultrell und interreligiös lebten. Das gegenseitige Milieu wurde schon funktioniert. Dank dem außerordenltlichen Wirken des Propheten wurde diese gemeisame Begegnung sachlich verwirklicht. Hier konnte man die gerechtige Interkulturalität verschiedener Rassen, Farben und Religionen merken. Das hat die meisten positiven westlichen Islamwissenschaftler kritisch behandelt.

"Der Prophet ist so zwangsläufig in eine politische Rolle hineingewachsen. Es zeigte im Lauf der Monate und Jahre, dass er in der Lage war, die gewaltigen Aufgaben, die an ihn herantraten, von Fall zu Fall zu meistern. Sogar Raubzüge und kriegerische Unternehmungen wußte er schließlich mit Geschick zu organisieren oder anzuführen, obwohl ihm persönlich das Kriegshandwerk nicht lag. "20

Schimmel hat deutlich bewiesen, dass der Prophet eine angemessene Verbindung zwischen Religion und Politik gewann. Er hat die allen irdischen Mitteln bedient, um einen unerschütterlichen islamischen Staat zu bauen. So gilt sie die Persönlichkeit des Propheten ein Vorbild der modernen Muslim, der sich mit der Politik besonders beschäftigt.

"Für einen Dualismus zwischen; guter geistiger und böser irdischer Sphäre gibt es im ursprünglichen Islam keinen

${ }^{19}$ Serauky,Eberhard. Geschichte des Islam. S.90.

${ }^{20}$ Paret, Rudi : Muhammad und der Koran. S.115. 
Das Prophetenbild in der modernen deutschen Literatur ein interreligiöses Diskurs

Dr. Tarek Mohammed Abo El-meilla Mhani

مجلة وادي النيل للاراسات والبحوث الإنسانية والاجتماعية والتربوية (مجلة علمية محكمة)

Raum, denn auch die Welt ist von Gott geschaffen und von Ihm dem Menschen untertan gemacht worden. "21

Sie erklärte auch, dass diese Besonderheiten des Propheten $\mathrm{zu}$ einer raschen Ausbreitung des Islam über die arabische Halbinsel führte. Das war nicht so im Fall der christlichen Botschaft, die Abwertung von der Welt als unvollkommen und mangelhaft ansah. Deshalb berücksichtigte Schimmel die Botschaft des Propheten als eine totale bessere Veränderung der Geschichte der Menschheit.

"Die rasche Ausbereitung des Islam über die arabische Halbinsel noch zu Lebzeiten des Propheten, aber noch mehr unvorstellbar rasche Ausdehnung des islamischen Reiches in den ersten hundert Jahren nach Muhammads Tode ließ keinen Zweifel zu, dass diese siegreiche Religion die wahre Religion war, und derjenige, der sie gepredigt hatte, der wahre, die endgültige Offenbarung bringende Prophet Gottes, der zu den Roten und Schwarzen, d.h. zu aller Welt gesandt war."22 " Auch die Theologen sehen in ihm die Tugenden sämtlicher vorhersehender Propheten verkörpert. Die Dichter wussten, dass er die Schönheit aller Propheten in ihm aufleichtet. ${ }^{23 "}$

\section{3- Die ethische Haltung Muhammads}

Um das sämtliche Bild des Propheten Muhammad im Werk von Schimmel $\mathrm{zu}$ thematisieren, soll die vorliegende Untersuchung die ethische Seite der Propheten entlarven. Hier habe ich eigentlich vor, einige verschiedene Züge der ethischen Handlungen des Propheten $\mathrm{zu}$ erhellen. Die erhabenen Moralen und Tugenden des Propheten werden im

${ }^{21}$ Schimmel. Und Muhammad ist Sein Prophet. S.47.

${ }^{22}$ Ebd.S.47.

${ }^{23}$ Schimmel, Annemarie: Die Zeichen Gottes, die religiöse Welt des Islam, München 1955, S.239. 
مجلة وادي النيل للاراسات والبحوث الإنسانية والاجتماعية والتربوية (مجلة علمية محكمة)

(ISSN : 2536 - 9555)

höchsten Maße hoch gepriesen. Seine Gegner haben diese Tatsache erkennbar bestätigt. Aus den Werken von Schimmel werde ich einige Beispiele der ethischen Seite des Propheten entnehmen.

"Die Propheten haben den Menschen nicht nur die Botschaft und die Rechtleitung Gottes gebracht, sondern ihnen auch ein gewisses Maß an Wisssen um Gott und Seine Name und Attribute vermittelt. Die dringlichste Aufgabe der Propheten bestand darin, die Realität dieses Lebens, seinen wahren Zweck und seine Bedeutung zu lehren. Da Gott jenseits der menschlichen Wahrnehmung und jenseits des menschlichen Begriffsvermögens steht, kam den Propheten die Aufgabe $z u$, solange ihre Aufgaben wahrzunehmen, die gehorsamsten,sorgfältigen, bewusstesten und disziplinertesten Menschen zu sein."24

Vorrangig ist der Prophet im wesentlichen Sinne ein Mensch, der schon eine göttliche Botschaft für die ganze Menschheit übernahm." Sprich : Ich bin nur ein Mensch wie ihr. Mir ist eingegeben worden, dass euer Herr ein einziger Gott ist.( Sura 18-110)25. Schimmel beschreibt den Propheten Muhammed als " das schöne Vorbild" und unterstreicht das Menschentum des Propheten.

"Muhammed hat niemals behauptet, irgendwelche übermenschlichen Fähigkeiten zu besitzen. Er wollte nichts als Diener sein, dem offenbart worden ist, und wenn man ihn aufforderte, Wunder zu vollbringen, so wies er auf den Koran hin- dass er ihn seinem Volk in klarer arabischer Sprache

${ }^{24}$ Gülen, M. Fethulla: Fragen an den Islam, 4.überarbeitete Auflage , Fontäne 2005. S.114.

${ }^{25}$ Sinngemäßige deutsche Übersetzung des Heiligen Koran. ArabischDeutsch.Zweite Auflage. Übersetzt von Prof. Dr. Moustafa Maher. Sprachliche Revision: Elsa Maher. Allgemeine Revision. A.Huber. Herausgeber: Oberster Rat für Islamische Angelegenheiten. Kairo 2007. S.635.- 
Das Prophetenbild in der modernen deutschen Literatur ein interreligiöses Diskurs

Dr. Tarek Mohammed Abo El-meilla Mhani

مجلة وادي النيل للاراسات والبحوث الإنسانية والاجتماعية و التربوية (مجلة علمية محكمة)

verkündet hatte, das war das einzige große Wunder seiner Laufbahn. Muhammad wusste und wurde durch die koranischen Offenbarungen immer wieder daran gemahnt, dass er nur ein Mensch war, dessen Vorzug darin bestand, dass ihm die Offenbarung zuteil geworden war. ${ }^{126}$

"Hier nimmt Muhammed in der Tat den Platz des vollkommenen Menschen ein. Gott schuf ein mikrokosmischen Wesen, den vollkommenen Menschen, durch den Sein Bewußtsein Ihm manifestiert wird. Der vollkommene Mensch ist der Geist, in dem alle Dinge ihren Ursprung haben; der geschaffene Geist Muhammeds ist damit ein Modus des ungeschaffenen göttlichen Geistes und das Medium, durch das Gott Seiner Selbst in der Schöpfung bewußt wird."27

Für Schimmel ist der Prophet Muhammad der vollkommenste Mensch und der Weg, der die göttliche Schöpfung versinnbildlicht hat. Deshalb sieht Schimmel, dass der Prophet eine vorbildliche Art und Weise der allen Muslimen ist. In der Muhammads Handlungen verkörpert sich die göttliche Offenbarung als eine praktische Prüfung. Er lehnt jegliche übermenschliche Kraft kategorisch an. Der Koran ist nur das ausschließende ewige Wunder des Propheten, dass die Größe und Wahrheit seiner Botschaft bewiesen hat.

"Muhammed selbst hatte Legenden abgelehnt und jeden Persönlichkeitskult verboten. Das einzige Wunder, das er beanspruchte, war, seinem Volk die Worte des Koran getreu mitgeteilt zu haben"28, "Im Islam jedoch wird in ganz besonderer Weise das Menschentum Muhammeds betont. ${ }^{129}$

\footnotetext{
${ }^{26}$ Schimmel: Muhammed, S.20

${ }^{27}$ Schimmel: Mystische Dimensionen, S.317.

${ }^{28}$ Ebd. S.303

${ }^{29}$ Bobzin,Hartmut: Mohammed. S,22.
} 
مجلة وادي النيل للاراسات والبحوث الإنسانية و الاجتماعية والتربوية (مجلة علمية محكمة)

(ISSN : 2536 - 9555)

Häufig behandelt Schimmel die Menschheit des Propheten und unterscheidet das von der Natur des Jesus. Das heißt, das Vorbild des Propheten Mohammed immer ist erhältlich, weil er ein Mensch ist, wie die Muslimen sind. Die Nachahmung des Propheten Muhammad soll das höchste Ziel aller Muslimen sein.

"Die Person des Propheten zum Medium der religiösen Erfahrung,obgleich das Zentrum des Islam phänomenologisch gesehen, nicht der Gesandte ist, der die göttliche Offenbarung im Koran brachte, sondern der Koran als direkte göttliche Offenbarung. Doch die Muslime fühlten, dass die Gestalt des Propheten notwendig war, um den Glauben in seinem legalen Aspekt aufrechtzuerhalten, wie in der zweiten Hälfte des Glaubensbekenntnisses angedeutet ist. ${ }^{130}$

Schimmel beschreibt auch eine wichtige Eigenschaft des Propheten, die ihn als $\boldsymbol{U} \boldsymbol{m} \boldsymbol{m i}$ darstellte. Sie sah besonders an, dass Muhammed als Ummi ein klar fundierter Beweis für die Bestätigung seiner Botschaft war.

"Zu den wichtigsten koranischen Aussagen über den Propheten gehört die Besonderheit ummi, das ihm in Sura 7, Vers 157-158 beigelegt wird. Es wird in der islamischen, vor allem der mystischen Traditionen als " des Lesens und Schreibens unkundig, Analphabet" aufgefasst. Für die Theologen und noch mehr die Mystiker des Islam war der Begriff ummi im Sinne von Analaphabet ein wunderbarer Beweis für die echte Inspiration des Propheten, für die Wahrheit seiner Sendung: wie hätte er, der nicht lesen und schreiben konnte, denn Kenntnis von so vielen Ereignissen der Vergangenheit und Zukunft haben können, wie sie im Koran aufgezeichnet sind? War nicht seine Qualität als ummi

${ }^{30}$ Schimmel: Mystische Dimensionen, S.304. 
Das Prophetenbild in der modernen deutschen Literatur ein interreligiöses Diskurs

Dr. Tarek Mohammed Abo El-meilla Mhani

مجلة و ادي النيل للاراسات والبحوث الإنسانية والاجتماعية والتربوية (مجلة علمية محكمة)

geradezu die Garantie dafür, dass der Koran Gottes Wort, seine reine unverfälschte Offenbarung war?"31

Die meisten westlichen Interpreter übten eine scharfe Kritik an dieser Eigenschaft des Propheten, aber Schimmel und die meisten islamischen Mystiker waren anderer Meinung. Goethe selbst hat diese Eigenschaft im wesentlichen Sinne gelobt und respektiert. Goethe hat einen großen Wert auf das gesprochene Wort mehr als auf das geschriebene Wort. ${ }^{32}$

"Dass Muhammed im Koran als Ummi bezeichnet wirdein meist als Analphabet erklärtes Wort- hatte nach Auffassung der Sufis eine tiefere Bedeutung. Denn nur ein Mensch, dessen Herz nicht von äußerer intellektueller Gelehrsamkeit verdorben war, sondern ein ebenso reines Gefäß dargestellt wie Maries Jungfräulicher Leib, konnte ein würdiges Gefäß für das göttliche Wort sein."33

Das Wissen des Propheten ist nicht aus einigen überlieferten Quellen geschöpft, sondern ist von Gott unmittelbar, makellos und ganz richtig, wie die Sura der Stern bestätigt. hat." Er ( Muhammad) spricht nicht nach Lust und Laune - Der Koran ist eine Offenbarung, die ihm von Gott eingegeben wird.(Verse:3.4)"134

Die Unweißheit des Propheten bekräftigt, dass er die Wahrheit verkündet, die ihm offenbart wird und nichts Falsches sagt." Mit solchem Wissen ausgerüstet, wird Muhammad dann als Begründer der verschiedenen Wissenschaftszweige betrachtet, und wie die Mystiker ihre

${ }^{31}$ Schimmel:Muhammad. S,36.

32 Siehe Mhani, Tarek: Ost- westliche Interkulturalität anhand der literarischen Werke von Goethe und Iqbal(Diss), 2010, S,89.

${ }^{33}$ Schimmel: Mystische Dimensionen, S.310.

${ }^{34}$ Sinngemäßige deutsche Übersetzung des Heiligen Koran, S.1121. 
مجلة وادي النيل للار اسات و البحوث الإنسانية و الاجتماعية والتربوية (مجلة علمية محكمة)

(ISSN : 2536 - 9555)

Traditionskette auf ihn zurückführen, so finden auch Kalligraphen und Historiker, Naturwissenschaftler und Mediziner ihr erstes großes Vorbild im Propheten. ${ }^{135}$

Schimmel hat sich ganz eingesetzt, um eine Tatsache $\mathrm{zu}$ bestätigen, dass Muhammad ein wahrer Prophet im Sinne des Wortes war. Es gelang ihr, das echte koranische Bild des Propheten zu veranschaulichen.Hier wird die Menschheit des Propheten besonders betont. Sein einziges Wunder ist der Koran, der als eine ewige Herausforderung für die ganze Menschheit gilt. Schimmel hat die Menschheit und die Unweißheit des Propheten des Lesens und Schreibens hoch respektiert.

"In allen Berichten wird seine Demut und Milde besonders hervorgehoben. Außerdem wird sein freundlicher Ernst in diesen Berichten betont und von der Tatsache berichtet, dass er nicht viel gelacht habe. Er habe jedoch ein gewinnendes Lächeln. Muhammads Rücksichtnahme auf Schwache und seine Liebenswürdigkeit wird gepriesen. Er schlug keinen Diener und keine Sklavin und keiner seiner Frauen, berichtet die Tradition." 36

Der Prophet gewann das höchste Vorbild der Moralen und Handlungen für die ganzen Muslimen. Er stellte die koranischen Vorschriften dar. Schimmel thematisierte die verschiedenen Aspekte seiner ethischen Haltung besonders aus mystischer Sicht in Einzelheiten.

"Muhammads liebevolle Zuneigung erstreckte sich auf alle Wesen. Kinderliebe war ein Ausdruck davon- er grüßte Kinder auf der Straße und spielte mit ihnen. Spätere Volksballaden erzählen in rührenden Versen, wie seine beiden Enkel Hasan und Husain beim Gebet" dem Großpapa Prophet" auf den Rücken geklettert seien, ohne daß er sichdurch die Jungen

${ }^{35}$ Schimmel: Muhammad. S,39.

${ }^{36}$ Ebd.S. 29 
Das Prophetenbild in der modernen deutschen Literatur ein interreligiöses Diskurs

Dr. Tarek Mohammed Abo El-meilla Mhani

مجلة وادي النيل للاراسات والبحوث الإنسانية والاجتماعية والتربوية (مجلة علمية محكمة)

habe stören lassen. Auch Tierliebe gehört zu seinen Eigenschaften. ${ }^{137}$

Muhammads Moralen sind als ein Wunder in der menschlichen Geschichte zu betrachten. Über ihn berichtete seine Gefährten, er ist eine Stütze für diejengen gewesen, die Hilfe brauchten. Er leistete diese Dienste niemals herablassend. Er war einfach gekleidet und lebte mit den Armen und war auch nicht begütert. Seine erhabene Moralen fanden überall in der Welt eine Resonanz, so dass viele Menschen aufgrund seiner maßgeblichen Ethik zum Islam bekehrt haben.

Ein anderer Aspekt der ethischen Haltung des Propheten ist die Frauenbehandlung. Im wesentlichen Sinne bringt der Islam der Frau eine sehr hohe Achtung entgegen. Immer plädiert er für eine völlig wahre Gleichberechtigung von Frauen und Männern. Im Islam stehen die Frauen auf dem festen Boden wie die Männer.

Sie repräsentierten die Hälfte der Gesellschaft. Ohne sie ist das Leben kategorisch absurd. Deshalb entählt das Leben des Propheten viele Hinweise auf die Frauenwürdigkeit. Mehrmals hat er den Muslimen empfohlen, die Frauen menschlich und liebevoll zu behandeln.

"Mit der Behandlung seiner Frauen, unter denen der Tradition nach durch aus nicht immer Einmütigkeit herrschte und die vor allem auf die junge A'ischa eifersüchtig waren, setzte Muhammad ein Beispiel für seine Gemeinde. Heiraten ist meine Sunna, dieses Wort wurde ihm frühzeitig zugeschrieben, und in der Tat hat der Islam kein zöbilatäres Ideal. Die islamischen Exegeten haben sich selbstverständlich mit dem Problem der Ehen des Propheten befasst, und während sie der Ansicht sind, dass Frauen im Allgemeinen

${ }^{37}$ Schimmel: Und Muhammad ist Sein Prophet. S.42. 
مجلة وادي النيل للار اسات و البحوث الإنسانية و الاجتماعية والتربوية (مجلة علمية محكمة)

(ISSN : 2536 - 9555)

den Mann von Gott ablenken können, ist dem nicht so im Falle des Propheten. Zudem symbolisieren seine Ehen, dass er kein Heiliger ist, der sich von der Welt zurückzieht, sondern jemand, der die Welt akzeptiert, in ihr lebt und sie eben dadurch heiligt." 38

Für Schimmel ist der Prophet eine Person, der seinen Frauen bei den Lasten des Haushalts behilflich war. Seine Liebe, Barmherzigkeit und Gerechtigkeit sind ein hervorragendes prophetisches Motto, das im Zeitleben des Propheten leicht zugänglich war. Er hat sich keineswegs von der Welt distanziert. Der Prophet hat die irdischen Mitteln bedient, um ein glückliches Leben mit seinen Frauen zu genießen.

"Die Liebe des Propheten zu seinen Frauen und zu seiner Tochter Fatima schloss ein völlig negatives Urteil aus. "39 Vor allem aber stand vor den Gläubigen der bekannte Ausspruch " Gott hat mirlieb gemacht von eurer Welt der Frauen und den Wolhgruch". Aus dem Leben des Propheten kann man viele Vorbilder dafür entnehmen.

"Die Ehe mit Hadiga stellte für Muhammad einen Wendepunkt in seinem früheren Lebensabschnitt dar. Mit ihr gewann er eine Lebensgefährtin, die unerschütterlicher Treue an seiner Seite stand und ihm in den schwierigsten Momenten einen sicheren Halt bot. Wesentlich war auch ihre geistige Aufgeschlossenheit, die sich nicht nur in einem wachen Verhältnis von Zeitfragen erwies, sondern auch in einer ausgeprägten Sensibilität für ideologische Spannungen bemerkbar machte. Sie bot ihm eine Partnerschaft, die ihm das Selbstbewußtsein stärkte, aber gleichzeitig einen tieferen Einblick in die Verhältnisse der Zeit ermöglichte. Die Bedeutung dieser Frau für die Herausbildung der ersten

${ }^{38}$ Schimmel. Muhammed. S.31.

${ }^{39}$ Schimmel: Und Muhammad ist Sein Prophet. S.45. 
Das Prophetenbild in der modernen deutschen Literatur ein interreligiöses Diskurs

Dr. Tarek Mohammed Abo El-meilla Mhani

مجلة وادي النيل للاراسات والبحوث الإنسانية والاجتماعية والتربوية (مجلة علمية محكمة)

islamischen Vorstellungen Muhammads kann keinsfalls überschätzt werden." 40

In diesem Zusammenhang darf man auch die hohe Achtung nicht vergessen, die der Prophet den Müttern zollte. Er betrachtet sie als den Weg zur Gottes Gnade.So empfohl er den Männern und Frauen, ihre Mütter besonders zu pflegen. " Das Muttermotiv spielt im Islam eine zentrale Rolle. Man hat darauf hingewiesen, dass das Wort rahma Barmherzigkeit, der gleichen arabischen Wurzel entstammt, wie rahim , Mutterscoss; es wäre daher durchaus möglich, von der Mutterliebe im weitesten Sinne zu sprechen.

Das Paradies liegt unter den Füßen der Mütter, sagt der Prophet, und der Mutter gebührt die nimmer endende Fürsorge des Menschen." 41

Es gelang Schimmel, dem tradierten verzerrten Frauenbild im Westen zu erwidern. Ihr Werk, Meine Seele ist Frau, war ein gutes Beispiel dafür. Sie hat schon bewiesen, dass es keine Abwertung der Frauen im Islam durch das Leben des Propheten gibt. Die verschiedensten Züge der Prophetenverehrung der Frauen fließen mehrmals im Werke Schimmel zusammen.

"Jedoch wäre es erstaunlich, wenn der Islam eine ausgesprochene frauenfeindliche Religion wäre, hat doch der Prophet Muhammad in einem oft kommentierten Wort gesagt:"Es wurden mir lieb gemacht von eurer Welt Frauen und Wohlgeruch, und mein Augentrost ist im Gebet". Die Frauen sind etwas Wohlduftendes: gut, tyyib, und Duft tib, gehören zur gleichen arabischen Wurzel. Man denke auch daran, dass die erste Gattin des Propheten, Khadidscha (gest.619), mit der er 25 Jahre in monogamer Ehe lebte, ihn

${ }^{40}$ Serauky, Eberhard: Geschichte des Islam. S.46.

${ }^{41}$ Schimmel. Meine Seele ist Frau, S.19. 
مجلة وادي النيل للار اسات و البحوث الإنسانية والاجتماعية والتربوية (مجلة علمية محكمة)

(ISSN : 2536 - 9555)

bei den unerhörten seelischen Schock, den die ersten Offenbarungen auslösten, unterstützte und tröstete. ${ }^{142}$

Schimmel hat den Islam im weitesten Sinne gut verstanden. Sie hat ein klares Ziel verfolgt, dem Westen ein richtiges Islambild zu überbringen. Sie war ganz bereit, alle Folgen dieser Haltung in Kauf zu nehmen. Deshalb kritisierte sie streng die Mystiker, die die Bewertung der Frauen im Islam verachtet haben.

"So sehr die islamischen Asketen auch die Frau als gefährliches Wesen schmähen mochten- einer absoluten Abwertung stehen die koranischen Versen entgegen, in denen immer wieder von frommen und gläubigen Männern und Frauen gesprochen wird, von den Musliminnen, die gleichen religiösen Pflichten haben wie die Männer der Gemeinde und ihren Lohn dafür erhalten werden. "143

Schließlich war Schimmel zum Erkenntniss gekommen, dass die Frauen im Islam eine hohe Wertung wie die Männer besaßen. Unablässig hat sie sich mit den strittigen Fragen der Frauen sachlich beschäftigt. Dehalb gewann sie ein unerschütterliches Selbstbewußtsein, die ganzen Rechte der Frauen im Islam zu entlarven. Durch das Leben des Propheten kamen die verschiedenen Züge zum Ausdruck, die das frauenfeindliche Bild katagorisch ausschloßen. Deshalb erlangten ihre Beiträge positive Wirkungen auf die westliche Haltung im Bezug auf das islamische Frauenbild.

Auf der anderen Seite hat Schimmel die hervorragende Verehrung der Muslimen des Propheten Muhammad in Einzelheiten rechtfertigt. Der Koran hat viele Versen, die den Muslimen eine unerhörte Hochachtung für den Propheten empfohlen hat. Für jeden Muslim ist die Leitung des Propheten bindend, weil er die Vorschriften des Islam im

${ }^{42}$ Ebd. S.19.

${ }^{43}$ Schimmel: Und Muhammed ist Sein Prophet ; S.45. 
Das Prophetenbild in der modernen deutschen Literatur ein interreligiöses Diskurs

Dr. Tarek Mohammed Abo El-meilla Mhani

مجلة وادي النيل للاراسات والبحوث الإنسانية والاجتماعية والتربوية (مجلة علمية محكمة)

weitesten Sinne praktiziert hat. Deshalb tritt die Verehrung des Propheten in den Vordergrund.

"Muhammad ist für die Muslime ein Vaterfigur, der verehrte Älteste der menschlichen Familie, in dem die Offenbarungen aller früher Propheten gipfeln, derjenige, dessen Beispiel man folgen muss, wie man dem Beispiel des gebliebten Vaters folgt. Seine Gegenwart wird erfahren, wenn der Segen Gottes über ihn herabgefleht wird; sein Name trägt segnende Kraft in sich; der Besuch seines Grabes in Medina hat Millionen von Menschen seelisch erhoben, und Dichter aller Zungen haben ihre Sehnsucht nach diesem Platz gesungen. Und wenn er durch die Jahrhunderte als Fürsprecher am Tage des Gerichts erscheint, dessen Liebe man sich verlassen kann, so sieht der moderne Politiker in ihm den Gründer einer Kultur, in der religiöse Hingabe und Wirken in der Welt unlösbar verbunden sind, einen politischen Führer, der Gleichheit und Brüderlichkeit gepredigt." 44

Für Schimmel war der Prophet der vollkommenste Mensch, der die ehrlichsten Tugenden und Besonderheiten besaß. Deshalb hat er als höchstes Vorbild für allen Muslimen bedient. Der Gott und seine Engel hat ihn auch sehr hoch gepriesen.

"Der Prophet ist als Barmherzigkeit für die Welten gesandt, und Gott und die Engel segen ihn. Denn er ist wahrlich von edler Natur. Mehrfach findet sich der Befehl: Gehorscht Gott und gehorscht Seinem Gesandten. Aus diesen und vergleichbaren Sätzen im Koran entwickelte sich bald eine weit über das Normalmaß hinausgehende Verehrung des Propheten, und kleine koranische Hinweise wurden im Laufe der Zeit zu wundersamen Erzählungen und Legenden

${ }^{44}$ Schimmel. Muhammd,S.7. 
مجلة وادي النيل للار اسات و البحوث الإنسانية والاجتماعية والتربوية (مجلة علمية محكمة)

(ISSN : 2536 - 9555)

ausgesponnen, die das Bild des historischen Bild mehr und mehr mit einem bunten Lichtschleier umwoben." ${ }^{145}$

Schimmel erklärte diesen Punkt in ihrem Werk - Und Muhammad ist Sein Prophet, Die Verehrung des Prophetenin der islamischenFrömmigkeit - in Einzelheiten. Die verschiedenen Züge dieser Verehrung besonders anhand der mystischen Kreisen wurden hier erkennbar. Sie versinnbildlichte ein allumfassendes Bild des Lebens von dem Propheten. Mit diesem Buch gelang ihr, dem tradierten westlichen Islambild zu erwidern.Daraufhin erreichte sie die Spitze der Interreligiösität durch ihre sachliche Beiträge des Propheten.

"Es versteht sich von selbst, dass die Verehrung des Propheten und das Interesse an selbst den kleinsten Details seines Wesens in dem Maße wuchs, wie sich die Muslime zeitlich von ihm entfernten. Immer mehr wünschten sie von seinem Wesen, seinem Aussehen, seinem Benehmen, um sicher $z u$ gehen, daß sie ihm richtig folgten. Die volkstümlichen Prediger taten viel dazu, die Gestalt des Propheten mit wunderbaren Farben auszumalen, und manchmal waren die orthodoxen Theologen wenig erbaut von ihren, wenn auch wohlgemeinten, Übertreibungen. ${ }^{146}$

\section{Ergebnisse}

Zum Abschluss meiner vorliegenden Untersuchung möchte ich die folgenden Erkenntnisse zusammenfassen:

- Ausdrücklich plädierte der Prophet Muhammad für den absoluten Monotheismus. Er hat die früheren Propheten verfolgt, an den Glauben an Einen Gott zu rufen. Für Schimmel ist der Monotheismus eine unerschütterliche Gemeinsamkeit der verschiedenen geoffenbarten Religionen.

\footnotetext{
${ }^{45}$ Ebd.S.21.

${ }^{46}$ Schimmel. Und Muhammad ist Sein Prophet, S.28.
} 
Das Prophetenbild in der modernen deutschen Literatur ein interreligiöses Diskurs

Dr. Tarek Mohammed Abo El-meilla Mhani

مجلة وادي النيل للاراسات والبحوث الإنسانية والاجتماعية والتربوية (مجلة علمية محكمة)

Deshalb respektiert der Islam diese Offenbarungsreligionen als verschiedene Wege, die richtig zum Gott führen können. Schimmel legte eine besondere Wertung auf den Monotheismus auf, weil er als die erste und unerläßliche Säule der Botschaft des Propheten gilt, der die Annäherung der anderen geoffenbarten Religionen rechtfertigt hat.

- Schimmel hat kritisch bewiesen, dass bereits der Prophet Muhammad in seiner Moschee in Medina einen Dialog mit den Juden und Christen führte. Es gelang ihm, Medina als eine interkulturelle und sowie interreligiöse Stadt aufzubauen. Die verschiedenen Rechte der medinensischen Mitbürger wurden sachlich garantiert, weil der Islam für eine klare Anerkennung aller Offenbarungreligionen plädiert hat. Der Prophet konnte erfolgreich zwischen der Religion und Politik vereinbaren, um ein interreligiöses Milieu durch den Dialog zu gründen. Die Verschiedenheiten der Rassen, Religionen und Kulturen wurden harmonisch verschmolzen.

-Schimmel hat die ethische Haltung des Propheten am schönsten ausgedrückt. Sein Menschentum tritt besonders in den Vordergrund. Durch die Menschheit des Propheten können die Muslimen ihre Hauptziele gut kennenlernen. Der Prophet lehrt, dass der Islam nicht die totale Abwertung der Welt verlangt. Der Mensch ist ein unweigerlicher Teil der Schöpfung deshalb respektiert der Islam die Würde aller Menschen. Am besten hat der Prophet den bestmöglichen irdischen Mitteln bedient, um das Leben richtig zu genießen. Daraufhin dient das menschliche Leben des Proheten allen Muslimen als Vorbild. Dehalb gelang es ihm, den Menschen seine Botscaft zu vermitteln.

- Mehrmals hat Schimmel betont, dass der Islam für einen wahren Respekt der Frauen plädiert hat. Prinzipell weden die ganzen Rechte der Frauen im Islam garantiert. Das westliche Frauenbild im Islam hat Schimmel besonders motiviert, um 
das Richtige und Sachliche zu suchen. Sie hat sich durch ihr Buch"Meine Seele ist Frau" ganz für eine neue Formnug des islamischen Frauenbilds im Westen eingesetzt.

- Das Leben des Propheten beinhaltet viele Hinweise auf die gute Behandlung seiner Frauen. Seine Leibe und Loyalität gegenüber Hadiga haben das verzerrte westliche Frauenbild ausgeschlossen. Er hat sich auch von dem Leben seiner Familie nicht zurückgezogen. Immer half er ihnen bei der schweren Haushalt. Ausdrücklich hat er den all Muslimen ständig empfohlen, die Frauen menschlich und geduldig zu behandeln. Die Bedeutung der Mütter ist keineswegs im Islam ignoriert. Die Rücksichtnahme der Mütter ist für allen Muslimen bindend.

-Ich hoffe, dass der Zweck dieses Beitrags erfüllt wird. Die Intrreligiösität wird durch das Propetenbild von Schimmels Werke sachlich funktioniert. Sie hinterlässt kritische, ostwestliche Untersuchungen im Bezug auf Respekt vor der Menschenwürde. Immer unterstreicht sie die gegenseitigen Gemeinsamkeiten zwischen verschiedenen Farben, Kulturen und Religionen. Schimmel legt die absoluten Verschiedenheiten zwischen Kulturen beiseite. Es gelang ihr, einen wissenschaftlichen und kritischen Weg $\mathrm{zu}$ den Annäherungen unterschiedlicher Völker und Nationen zu geben. Das ist die dringlichste Botschaft der ost-westlichen Vertreter, die eine friedliche Welt für alle Menschheit zu erweisen suchen. Das macht die Frage der Interkulturalität dringend in unserer heutigen Zeit.

\section{Literaturverzeichnis}

- Bobzin, Hartmut: Muhammad. Verlag C.H. Beck OHG, München2005.

- Bobzin, Hartmut: Der Koran. C.B. Verlag. München 2007. 
Das Prophetenbild in der modernen deutschen Literatur ein interreligiöses Diskurs

Dr. Tarek Mohammed Abo El-meilla Mhani

مجلة وادي النيل للاراسات والبحوث الإنسانية والاجتماعية و التربوية (مجلة علمية محكمة)

- Gülen, M. Fathulla: Fragen an den Islam, 4 überarbeitete Auflage, Fontäne Verlag 2005.

- Khaled, M. Abu-Hattab: Martin Luther zwischen Islam und Reformation, Gedanken zur Reformation in ihren Beziehungen zum islamischen Diskurs. Kairo 2010.

- Krämer, Grunden: Geschichte des Islam. C.B. Verlag. München 2005.

- Mhani, Tarek: Ost-westliche Interkulturalität anhand der literarischen Werke von Goethe und Iqbal (Diss.), 2010.

- Moussa, M. Youssef: Islam und dessen Notwendigkeit für die Menschheit. Übersetzt von Elisabeth Khorshed. Oberstenrates für Islamische Angelegenheiten 2004.

- Paret, Rudi: Mohammed und der Koran, W. Kohlhammer, neunte Aufl., Stuttgart 2005.

- Rückert, Friedrich: Der Koran. hrsg. v.Hartmut Bobzin mit erklärenden Anmerkungen von Wolfdietrich Fischer, Ergon Verlag, Würzburg 1955.

Schimmel , Annemarie: Die Zeichen Gottes- die religiöse Welt des Islam, München 1955.

- Schimmel, Annemarie: Meine Seile ist Frau, das Weibliche im Islam. Kösel 1955.

- Schimmel, Annemarie: Und Muhammad ist Sein Prophet, die Vereherung des Propheten in der islamischen Frömmigkeit, Eugen Diederiches Verlag. 2.verb. Aufl. München 1989.

- Schimmel, Annemmarie: Mystische Dimensionen des Islam. Frankfurt a. Main u.a. 1995. 
مجلة وادي النيل للاراسات و البحوث الإنسانية والاجتماعية والتربوية (مجلة علمية محكمة)

(ISSN : 2536 - 9555)

- Schimmel, Annemarie: Muhammad. Deutsche Bibliothek, München 2000.

- Schimmel, Annemarie: Speigelungen des Islam, Ed.q. Berlin 2002.

- Serauky, Eberhard. Geschichte des Islam, EntstehungEntwicklung und Wirkung von den Anfängen bis zur Mitte des 20. Jahrhunderts, Gulde Druck, GmbH, Thübingen 2003.

- Sinngemäßige deutsche Übersetzung des Heiligen Koran. Arabisch-Deutsch. Zweite Auflage. Übersetzt von Prof. Moustafa Maher. Sprachliche Revision: Elsa Maher. Allgemeine Revision: Ali Huber. Herausgegber: Oberster Rat für Islamische Angelegenheit. Kairo 2007. 